\title{
Coarse Tracking System Identification of Compound Axis Control System based on the Measured Operation Data
}

\author{
Sen $\mathrm{Bu}^{1, a}$, Shengli $\mathrm{Qu}^{2, \mathrm{~b}}$ and Bo Qian ${ }^{3, \mathrm{c}}$ \\ ${ }^{1}$ No. 2, South Taibai Road, Xi'an City, Shaanxi Provence, China \\ ${ }^{2}$ No. 2, South Taibai Road, Xi'an City, Shaanxi Provence, China \\ ${ }^{3}$ No. 2, South Taibai Road, Xi'an City, Shaanxi Provence, China \\ asbu1314@163.com, bslqu@mail.xidian.edu.cn, cbqian@mail.xidian.edu.cn
}

\begin{abstract}
Keywords: coarse tracking, compound axis, difference, identification
Abstract. The coarse tracking system of compound axis control system has been determined. But the deep understanding of the coarse tracking system can only be analyzed by the measured operation data. Thus the linear model of the coarse tracking system can be identified only by the measured data. In this paper, we put forward the zero order, first order and second order differential treatment of the measured operation input and output data. By comparing the dynamic indexes of the unit step response of the identification model, the minimum error function and the simulation error, the best matching model is the second order system and second order difference.
\end{abstract}

\section{Introduction}

Optoelectronic tracking technology has been widely used in many fields, such as scientific research, precision measurement aerospace. And put forward more and more high request [1].Compound axis control system can meet the requirements.

Compound axis control system is a follow-up system, which is used to reduce the difference between the target direction and the tracking axis [2], it can greatly improve the tracking precision and response frequency of the photoelectric tracking system. Compound axis control system is composed of two main track frame and adjustable mirror (Fast Steering Mirrior, FSM) tracking structure of two axis is mounted on it[3].Therefore Compound axis control system is usually composed of two control systems, that are low bandwidth coarse tracking system and high bandwidth precision tracking system . As is shown in Fig. 1[4].The coarse tracking system has a larger working range and the precise tracking system has a small working range.

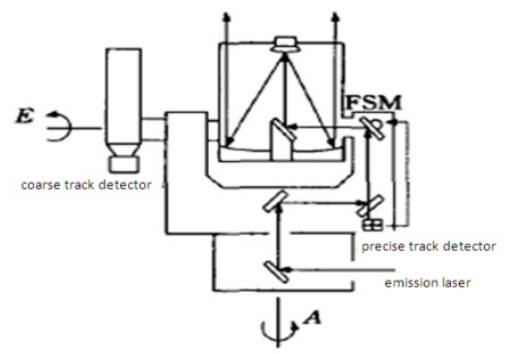

Fig. 1 Structure of compound axis control system

\section{Analysis of the Measured Operation Data and Multiple Difference}

Because the input and output data for coarse tracking system identification is the measured operation data, as is shown in Fig. 2. The input and output data is slow changing signal. From the point of view of identification, the measured operation input and output data can not fully excite the system, Therefore the precision of identification model is not high. 


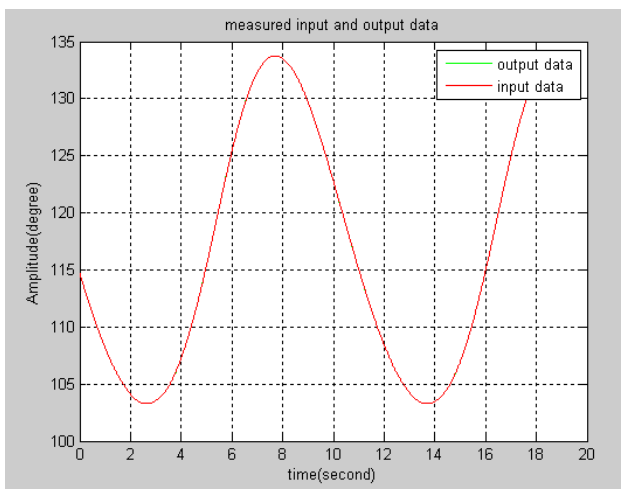

Fig. 2 The measured operation data

Thus we put forward a kind of solution that the measured operation data is processed by multiple difference.after multiple difference, the input and ouput data is changed into excitation signals, The system model can be fully excited. We can improve the precision of the identification model.

\section{Identification Results of Multiple Difference}

\section{Model Identification and Indexes Calculation.}

We identificate the parameters of coarse tracking system of the compound axis control system. We can only see a sinusoidal and asymmetry line in Fig. 2, This is because the input and output data is similar under the coordinate axis. Now we draw the input and output error, as is shown in Fig. 3.

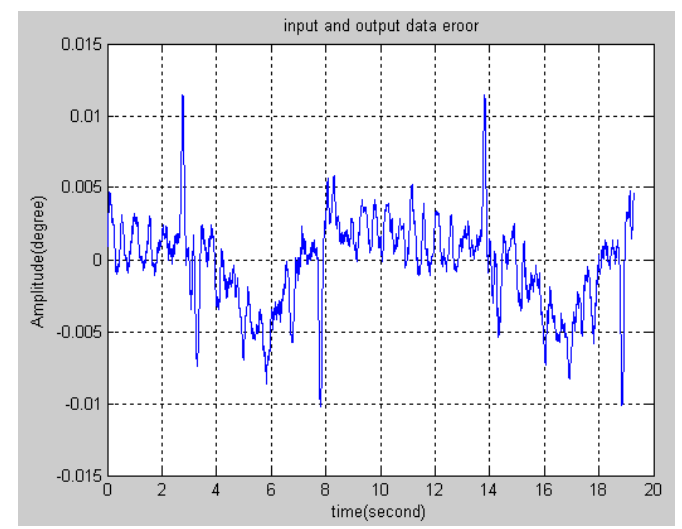

Fig. 3 The error of the measured input and output data

We assume the closed loop transfer function mode as the second order system of coarse tracking system of the compound axis control system. As is shown in Eq. 1.

$$
G(\mathrm{z})=\frac{b_{1} z^{-1}+b_{2} z^{-2}}{1+a_{1} z^{-1}+a_{2} z^{-2}}
$$

$a_{1}, a_{2}, b_{1}, b_{2}$ are identification parameters.

We can use the least squares method to identify the above parameters and to do the zero order, first order and second order difference of the measured operation of the input and output data.

The second order system and zero order difference:

The transfer function of the closed loop discrete system is:

$$
G(z)=\frac{0.06088 z-0.01728}{z^{2}-1.94 z+0.9838} \text {. }
$$


The transfer function of the closed loop continuous system is:

$$
G(s)=\frac{3.963 s+441.2}{s^{2}+1.629 s+440.9} .
$$

According to the minimum error function formula, $J=(\mathrm{Y}-U \hat{\theta})^{T}(\mathrm{Y}-U \hat{\theta}), J$ is the value of the minimum error function, $\mathrm{Y}$ is output observation data, $U$ is the input observation data, $\hat{\theta}$ is parameter estimation.

We can calculate the value of the minimum error:

$J_{0}=0.015$

The second order system and first order difference:

The transfer function of the closed loop discrete system is:

$G(z)=\frac{0.1053 z-0.04868}{z^{2}-1.726 z+0.7826}$

The transfer function of the closed loop continuous system is:

$G(s)=\frac{8.641 s+641.6}{s^{2}+24.51 s+642.6}$

The value of the minimum error:

$J_{1}=1.042 * 10^{-4}$.

The second order system and second order difference:

The transfer function of the closed loop discrete system is:

$G(z)=\frac{0.1081 z+0.09747}{z^{2}-0.1957 z-0.5452}$.

The transfer function of the closed loop continuous system is:

$$
G(s)=\frac{13.28 s^{2}+61.18 s+1366000}{s^{3}+104.2 s^{2}+102100 s+1721000} .
$$

The value of the minimum error :

$$
J_{2}=6.139 * 10^{-5} \text {. }
$$

We carry out the unit step response of the closed loop continuous transfer function. As is shown in Fig. 4. 


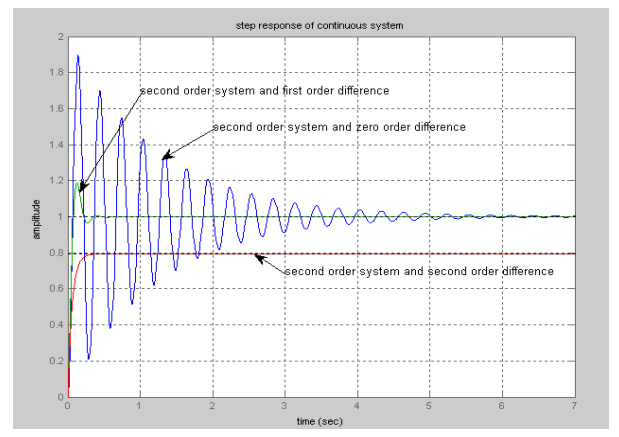

Fig. 4 Unit step response of the identification models

We can see from Fig. 4 , second order difference system has large steady-state error.Thus we need to multiply the proportional coefficient of the closed-loop transfer function of the continuous system to eliminate the steady-state error.

After correction of the proportion coefficient of the second order difference model of the second order system. The transfer function of the closed loop discrete system is:

$$
G(z)=\frac{0.1362 z+0.1229}{z^{2}-0.1957 z-0.5452}
$$

The transfer function of the closed loop continuous system is:

$$
G(s)=\frac{16.74 s^{2}+77.12 s+1721000}{s^{3}+104.2 s^{2}+102100 s+1721000} .
$$

The value of the minimum error:

$$
J_{2^{\prime}}=6.245^{*} 10^{-5} \text {. }
$$

The unit step response of the identification models is shown in Fig. 5.

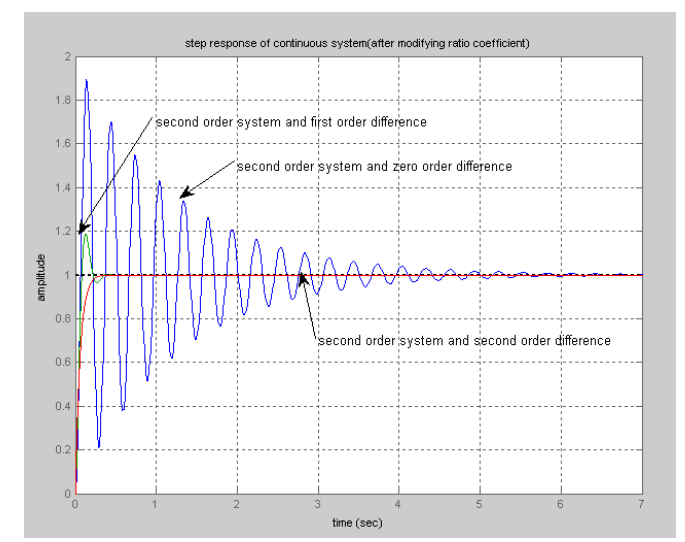

Fig. 5 Unit step response of the identification models (modified the coefficient)

We calculate the dynamic indexes of unit step response of each model:

The rise time $t_{r}$, adjusting time $t_{s}$ and the overshoot $\sigma \%$ of unit step response are :

The second order system and zero order difference: as is shown in Eq. 14. 


$$
\begin{aligned}
& t_{r}=0.075 \mathrm{~s} \\
& t_{s}=3.606 \mathrm{~s} \\
& \sigma \%=89.5 \% .
\end{aligned}
$$

The second order system and first order difference: as is shown in Eq. 15.

$$
\begin{aligned}
& t_{r}=0.081 \mathrm{~s} \\
& t_{s}=0.197 \mathrm{~s} \\
& \sigma \%=18.7 \%
\end{aligned}
$$

The second order system and second order difference: Because the second order difference step response of the second order system is monotonic increasing curve, there is no overshoot, Only concerned about its adjusting time. As is shown in Eq. 16.

$$
t_{s}=0.178 s \text {. }
$$

\section{Simulation Error Calculation}

We use the measured input data as the input of the identification model for simulation compared with the measured operation output data. As is shown in Fig. 6.

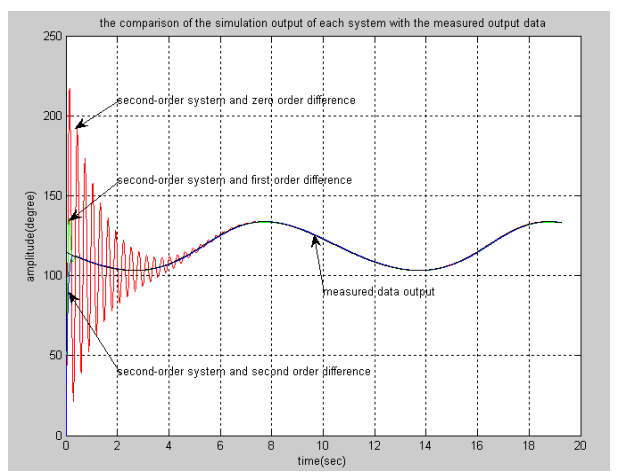

Fig. 6 Identification model simulation output and the measured output

Enlarge Fig. 6.As is shown in Fig. 7.

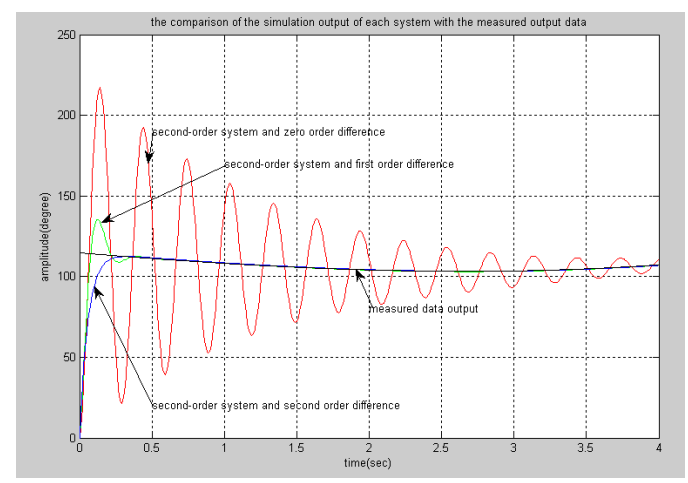

Fig. 7 Identification model simulation output and the measured output 
We need to calculate the square sum $\sigma^{2}$ of the difference between the simulated output and the measured operation output data.

The second order system and zero order difference : $\sigma_{0}^{2}$ is shown in Eq. 17.

$$
\sigma_{0}^{2}=4.146 * 10^{5}
$$

The second order system and first order difference : $\sigma_{1}^{2}$ is shown in Eq. 18.

$$
\sigma_{1}^{2}=4.420 * 10^{4}
$$

The second order system and second order difference: $\sigma_{2}^{2}$ is shown in Eq. 19.

$$
\sigma_{2}^{2}=4.640 * 10^{4}
$$

\section{Evaluation of Identification Results}

By least squares method, we identify the closed loop transfer function of the coarse tracking system of the compound axis control system. By means of differential operation of the measured operation input and output data,we obtain three second order systems, By comparing the dynamic indexes of the unit step response, such as rise time $t_{r}$, adjusting time $t_{s}$, and overshoot $\sigma \%$. And by comparing the minimum error function $J$, last we use the measured input data as the input of the identification model for simulation, and calculate the simulation error.

For mathematical model of the second order difference of the second order system, although its simulation error is larger than mathematical model of the first order difference of the second order system, in other indexes, the second order difference model is more advantageous than the first order difference model. Thus the best closed-loop transfer function is the model of the second order difference of the second order system. Therefore the best mathematical model of the coarse tracking system of the compound axis control system is Eq. 11 and Eq. 12.

\section{Conclusions}

Through multiple difference operation of the measured operation data, we can identificate the linear part of the coarse tracking system. It turns out that multiple difference does help to identify more accurate mathematical model. Thus from Fig. 6, the simulation curve of the second order difference and the second order system and the curve of actual output match well. From Fig. 3, There are nonlinear factors in the coarse tracking system. The identification is only for the linear part of the coarse tracking system. As for the nonlinear part, we will study the factors in the future.

\section{References}

[1] Jiaguang Ma, Tao Tang: Infrared and Laser Engineering. Vol. 42 No. 1 (2013) (In Chinese)

[2] Yufang Yue, Xiaogang Xie and Jianzhu An: Infrared and Laser Engineering. Vol.45 No.S1 (2016) (In Chinese)

[3] Xiulin Yang, Peiguo Lu,Xiaoqiang Liu and Xiaodong Liang: LASER \& INFRARED. Vol.45 No.8 (2015) (In Chinese)

[4] Shaojin Chen, Hongke Wang, Leigang Zheng and Bo Yang: Fire Control \& Command Control. Vol.37 No.4 (2012) (In Chinese) 
[5] Tingxia Liu: the research of Compund-axis Servo Control Technique of O-E Tracking System [D].Changchun: Chinese Academy of Science. 2005. (In Chinese)

[6] Feng Ding: New Theory of System Identification[M]. Beijng: Science Press, (2013), pp 199-201 (In Chinese)

[7] Shengsuo Niu, Da Zhang, Zhirui Liang and Xiaodi Huo: Power System Protection and Control.Vol.42 No.11 (2014) (In Chinese)

[8] Guihua Tan: Harmonic state estimation of power system based on total least squares and its error analysis[D]. Chongqing: Chongqing University, (2009) , pp 18-25 (In Chinese) 